\title{
Política de competencia y productividad: más allá de la calidad institucional y la apertura*
}

\author{
Joan-Ramon Borrell y Mara Tolosa \\ Instituto de Economía Aplicada (IREA) \\ Departamento de Política Económica \\ Universitat de Barcelona*
}

\section{Resumen}

Este trabajo estima el impacto de las políticas de competencia sobre la productividad de los países. La evidencia comparada internacional muestra que la calidad institucional y la apertura comercial de los países son factores que impulsan el output por ocupado y la productividad total de los factores. Aunque es difícil separar el impacto particular de la política de competencia del impacto de la calidad institucional en general, en este trabajo hemos estimado el efecto positivo y significativo de la política de competencia sobre el output por ocupado, y a niveles similares de calidad institucional, sobre la productividad total de los factores.

Palabras clave: competencia, productividad, instituciones, apertura.

Clasificación JEL: D7, L4, O4.

\begin{abstract}
This paper estimates the impact of antitrust effectiveness on productivity. The cross-country evidence shows that institutional quality and openness are drivers of the output per worker and of total factor productivity. Although it is difficult to separate out the effect of competition policy with respect to institutional quality controls, in this paper we estimate the positive and significant impact of antitrust effectiveness on output per worker, and for similar institutional quality, also for total factor productivity.
\end{abstract}

Keywords: antitrust, productivity, institutions, openness.

JEL classification: D7; L4; O4.

\section{Introducción}

Los avances de las últimas dos décadas en los trabajos de economía industrial teórica y empírica señalan que la competencia impulsa el crecimiento de la productividad y la competitividad a través de las tres vías que indican Aghion y Schankerman (2004): (1) la competencia fuerza a las empresas ya instaladas en los mercados

\footnotetext{
* Agradecemos la ayuda del Ministerio de Industria, Comercio y Turismo (DG de Política para la Pequeña y Mediana Empresa), y los comentarios de los miembros del Grupo de Investigación en Políticas Públicas y Regulación Económica (GPRE), en particular de Germà Bel, Joan Calzada, Christian Duran, y también de George Symeonidis a una versión preliminar de este trabajo. El trabajo se ha beneficiado de la investigación conjunta con Juan Luis Jiménez en otros manuscritos. Los errores u omisiones corresponden en exclusiva a los autores.
} 
a reducir sus costes de producción a través de mejoras en los procesos internos de producción física de bienes en las plantas productivas, en los procesos de coordinación entre centros de negocios, y en los procesos de prestación de servicios a los clientes; (2) la competencia entre las empresas ya instaladas hace que exista una continua reasignación de las cuotas de mercado desde las empresas menos dinámicas y productivas hacia las empresas más innovadoras; (3) la competencia permite la entrada de nuevas empresas más dinámicas y competitivas que las ya existente, y la entrada neta de empresas es una fuente de gran dinamismo y competitividad.

A pesar de este consenso académico, existe escasa evidencia empírica que cuantifique el impacto de la política de competencia sobre la productividad. De hecho, son también escasos los estudios que muestran que la política de competencia tenga un impacto significativo sobre la rivalidad en los mercados.

Hasta este momento existen trabajos que han intentado cuantificar el efecto de la política de competencia a partir de la evidencia internacional comparada a nivel de país. Asimismo, existen trabajos que utilizan evidencia de carácter sectorial para evaluar el impacto de la política de competencia sobre la productividad, los márgenes empresariales o los costes.

Este trabajo utiliza datos comparativos entre países para estimar el impacto de la política de competencia sobre el ouput por ocupado y la productividad total de los factores mediante técnicas de variables instrumentales. A diferencia de estudios anteriores en los que no había sido posible separar el efecto de la política de competencia del impacto de la calidad institucional de los distintos países, este trabajo muestra que es posible inferir un efecto significativo de la política de competencia sobre el output por ocupado, e incluso sobre la productividad total de los factores. De esta manera, muestra que las diferencias de productividad entre países se explican por políticas concretas que van más allá de las que favorecen un entorno de mayor calidad institucional y de mayor apertura exterior.

Tras esta breve sección introductoria, a continuación el trabajo se estructura en cuatro secciones más. En la sección 2 se revisa la literatura sobre el tema. En la sección 3 se presentan los datos y la estrategia empírica. En la sección 4 se muestran los resultados. Y la sección 5 concluye.

\section{Revisión de la literatura}

Existen dos tipos de trabajos empíricos publicados hasta el momento que intentan estimar los efectos de las políticas de competencia. Por un lado, unos estudios explotan la información comparativa entre países sobre las diferencias en la efectividad de la política de competencia y variables de resultados de crecimiento, productividad o inflación. Por otro lado, otros estudios utilizan evidencia de carácter sectorial para dilucidar el efecto de la política de competencia sobre los márgenes precio-coste, la concentración empresarial, la productividad y los salarios. 
De entre los estudios que utilizan evidencia a nivel de país destacan los siguientes. Hayri y Dutz (1999) estiman el impacto de la diferencia en la efectividad de la política de competencia sobre el crecimiento de los países a partir de ecuaciones de crecimiento. Encuentran un impacto positivo y significativo de la política de competencia sobre el crecimiento. Ahora bien, la inferencia no está corregida por el posible carácter endógeno de la política.

Krakowski (2005) estudia el efecto de la política de competencia sobre la intensidad de la rivalidad y la competencia en los mercados, y de ésta sobre el producto nacional bruto per capita utilizando regresiones múltiples y datos a nivel de país mediante técnicas de ecuaciones simultáneas robustas a la presencia de endogeneidad. Este trabajo muestra la correlación entre la efectividad de la política de competencia, la intensidad de la competencia en los mercados, y el producto per cápita. Asimismo, estima un impacto positivo y significativo de la política de competencia sobre la rivalidad en los mercados, y cómo la rivalidad impulsa el producto per capita. Con todo, el trabajo no muestra si los instrumentos utilizados para identificar el sistema de ecuaciones son válidos y fuertes. A diferencia de lo que es habitual en la literatura que mide el efecto de las políticas sobre el crecimiento y la productividad, se toman como variables exógenas que identifican el sistema tanto la calidad y efectividad de las políticas públicas, como el grado de apertura comercial ${ }^{1}$.

Voigt (2006) estudia el impacto de las diferencias en las leyes de competencia de distintos países sobre la productividad total de los factores. También encuentra un impacto positivo y significativo de la política de competencia sobre la productividad total de los factores. Ahora bien, la estimación no es robusta a la inclusión de variables que tienen en cuenta las diferencias en la calidad de las políticas públicas entre los distintos países. Así pues, Voigt (2006) no es capaz de separar el efecto general de la calidad de las políticas públicas, del efecto concreto de la política de competencia sobre la productividad. Además, la estimación no es robusta a la posible endogeneidad de la política de competencia.

Borrell y Tolosa (2008) realizan la primera estimación robusta a la presencia de políticas endógenas. Identifican de esta manera un impacto positivo y significativo de las políticas de competencia y apertura comercial sobre la productividad total de los factores. Ahora bien, no discuten en qué medida los resultados son robustos a la inclusión de variables que controlen por las diferencias en la calidad general de las políticas públicas en los países estudiados.

\footnotetext{
${ }^{1}$ Estos estudios incluyen los que estiman los efectos de las políticas y regulaciones sociales (BESLEY y CASE, 2000), de las políticas monetarias y fiscales (PERSSON y TABELLINI, 2003), de reforma regulatoria (AI y SAPPINGTON, 2002), y de desregulación (DUSO y RÖLLER, 2003, y DUSO, 2005). También los que estudian el impacto de las políticas de apertura comercial como por ejemplo DOLLAR (1992), SACHS y WARNER (1995), BEN-DAVID (1993) y EDWARDS (1998). Y otros que han evaluado el impacto de la apertura medida como el peso del comercio exterior respecto al PIB en ecuaciones de crecimiento: FRANKEL y ROMER (1999); ACEMOGLU, JOHNSON y ROBINSON (2001); RODRIK, SUBRAMANIAN y TREBBI (2002) y SACHS (2003).
} 
Przybyla y Roma (2005) estudian el impacto de la intensidad de la competencia sobre la inflación utilizando tanto datos a nivel de país como datos sectoriales. El grado de rivalidad en los mercados de productos, en particular medida a través de los márgenes precio-coste, reduce la inflación media a largo plazo. En este trabajo no se discute si los resultados son robustos a la posible endogeneidad de las variables estudiadas.

Por otra parte, entre los estudios que utilizan evidencia sectorial destacan los siguientes. Symeonidis (2000) estudia el efecto de la política de competencia sobre los márgenes precio-coste y la concentración sectorial en el Reino Unido. En un trabajo posterior Symeonidis (2008) analiza el efecto de la política de competencia sobre la productividad también en el Reino Unido. En ambos trabajos se tienen en cuenta los problemas de simultaneidad. Para ello, se utilizan datos de las industrias manufactureras entre 1954-1973. Este autor encuentra que la introducción de las leyes de cartel en 1950 intensificó la competencia en precios en las industrias manufactureras previamente cartelizadas, pero por el contrario, conllevó un aumento de la concentración sectorial. Asimismo, encuentra evidencia de un efecto negativo de la colusión sobre la productividad del trabajo.

Kee y Hoekman (2007) utilizan un rico panel de datos sectoriales correspondientes a 42 países durante 19 años para mostrar que la aprobación y aplicación de leyes de competencia no tiene impacto directo sobre los márgenes precio-coste, aunque sí tienen impacto positivo sobre el número de empresas en el mercado, y de esta forma indirectamente sobre la rivalidad y los márgenes.

Por el contrario, McCloughan, Lyons y Batt (2007) utilizan un panel de datos sectoriales para los países de la OCDE durante cuatro años y encuentran que aquellos países que tienen políticas de competencia más efectivas tienen márgenes precio-coste medios sectoriales menores.

Este trabajo se enmarca dentro de la literatura que utiliza evidencia comparativa a nivel de país. Pretende discutir utilizando técnicas robustas a la presencia de endogeneidad en qué medida las diferencias en la efectividad de las políticas de competencia tiene un impacto sobre el output por ocupado y la productividad total de los factores más allá de las diferencias en la calidad institucional y la apertura de los países de la muestra.

\section{Método para medir el efecto de las políticas}

El trabajo seminal de Hall y Jones (1999) inicia la literatura empírica que estudia el efecto de las políticas sobre la productividad. Según estos autores, un conjunto de aspectos históricos y geográficos observables de cada país influyen en la productividad exclusivamente a través de su impacto sobre las políticas y el medio institucional del país. Las medidas que ellos utilizan son un indicador de la calidad institucional (políticas anti-despilfarro o Government Anti-Divertion Policies, GADP) del país, y un indicador de política comercial (años de apertura comercial o years 
open, YRSOPEN). Estos autores encuentran que ambas explican una parte considerable de las diferencias de productividad entre países.

Las contribuciones empíricas que siguen a Hall y Jones (1999) han usado indicadores similares que intentan recoger en qué medida el ambiente institucional alienta las actividades económicas productivas. Acemoglu, Jonson y Robinson (2001) se centran en la protección del riesgo de expropiación por parte del gobierno (uno de los componentes del indicador de calidad institucional o políticas antidespilfarro usado por Hall y Jones, 1999); por su parte, Easterly y Levine (2003) utilizan un indicador más amplio que mide el «buen» ambiente institucional.

En términos formales, la propuesta de Hall y Jones (1999) se puede reescribir de la siguiente manera. Supongamos que denominamos $q$ al vector que contiene el resultado de un conjunto de países, por ejemplo medido a través del output por ocupado o la productividad total de los factores. Nuestro interés reside en identificar y estimar el efecto que tienen las políticas (calidad institucional o políticas antidespilfarro, política comercial, y política de competencia) sobre este vector de resultado tal y como muestra las ecuaciones 1 y 2 , donde estas políticas vienen recogidas en una matriz $\mathbf{S}$.

$$
\begin{aligned}
& q=\mathrm{f}\left(\mathrm{X}, \mathbf{S} ; \theta_{1}\right)+\varepsilon_{1} \\
& \mathbf{S}=\mathrm{g}\left(\mathrm{X}, \mathrm{Z} ; \theta_{2}\right)+\varepsilon_{2}
\end{aligned}
$$

Por su naturaleza, las políticas son endógenas: no sólo los países con mejores políticas tienen mayor productividad, sino que los países con mayor productividad son aquellos que seleccionan mejores políticas. Por ello, para identificar y estimar de forma robusta el efecto de las políticas sobre la productividad necesitamos especificar un sistema de ecuaciones en el que la productividad venga explicada por un conjunto de variables exógenas incluidas en la matriz X y la matriz $\mathrm{S}$ de políticas endógenas. Y a su vez, necesitamos especificar una ecuación en la que cada una de las políticas endógenas sea a su vez explicada por la matriz de variables exógenas $(X), y$ un conjunto de variables instrumentales exógenas que determinen la selección de las políticas por parte de los países pero no directamente la productividad (Z).

En este trabajo partiremos de los modelos propuestos por Hall y Jones (1999), y Persson y Tabellini (2003) en los que son los datos los que nos dicen si variables geográficas (como la latitud y otras) o históricas (como la colonización y otras) afectan a la productividad directamente, y por ello forman parte de las variables exógenas $(\mathrm{X})$, o bien sólo afectan a la productividad indirectamente a través de las políticas, y por ello forman parte de la matriz de instrumentos (Z).

\section{Datos y variables}

La mayoría de las variables utilizadas en nuestras estimaciones provienen de la base de datos de Persson y Tabellini (2003) y seguimos las notaciones utilizadas por 
estos autores. La base consta de datos de 85 países definidos como democracias de acuerdo al indicador de Freedom House que clasifica lo países en libres, semi-libres y no-libres. La muestra considera los países que entran en las primeras dos categorías. Dada la disponibilidad de nuestros datos referidos a la política de competencia, la muestra con la que finalmente trabajamos queda restringida a 52 países. En general, las variables se toman en promedio para el período 1990-1998, o bien para una cantidad de años menor dependiendo de la disponibilidad de datos.

La variable de desempeño o resultado de los países $(q)$ es el output por ocupado (YL) y la productividad total de los factores (A) medida para el año 1988. Estas variables proceden de los cálculos de Hall y Jones (1999) en el que se hace un ejercicio de inferencia del capital físico acumulado en los distintos países, y del capital humano, para obtener de esta manera una estimación de la productividad total de los factores.

Replicaremos en primer lugar para la muestra reducida de 52 países la estimación de Persson y Tabellini (2003) del efecto de la calidad institucional o políticas anti-despilfarro (GADP) y de apertura comercial (YRSOPEN) sobre la productividad. Y a continuación añadiremos a su especificación la política de competencia (COMP_EF).

La variable (GADP) muestra la percepción sobre la intensidad con la que el entorno económico e institucional promueve la expansión del producto y evita el mal uso de los recursos (depilfarro) entre 1986 y 1995. Se trata de una variable de síntesis de 5 indicadores: ley y orden, calidad de la burocracia, corrupción, riesgo de expropiación, y repudio de contratos y obligaciones por el gobierno. Este índice aproxima lo que Hall y Jones (1999) llaman la «infraestructura social».

La apertura al comercio exterior (YRSOPEN) se mide a través del índice calculado en Sachs y Werner (1995) que ofrecen Hall y Jones (1999). Muestra la fracción de años que la economía de cada país cumplió con un conjunto de requisitos para calificarla como economía abierta al comercio con el exterior entre 1950 y $1994^{2}$.

Para la efectividad de la política de competencia (COMP_EF) contamos con la medida elaborada por World Economic Forum y publicada en el Word Competitiveness Report (Porter et al., 2000) que recoge las respuestas de personas de negocios de 75 países a la siguiente pregunta: «¿la política antitrust en su país es $1=$ laxa y no efectiva en promover la competencia, 7 = efectivamente promueve la competencia)?». Tal y como ya hemos indicado, el proceso de emparejar los datos de Persson y Tabellini (2003) con esta variable restringe nuestra muestra a 52 países.

\footnotetext{
${ }^{2}$ Los requisitos son cinco: (1) arancel medio superior al 40\%, (2) barreras no comerciales que cubren en promedio a más del $40 \%$ de las importaciones, (3) sistema económico socialista, (4) monopolio estatal de exportaciones, (5) diferencial de tipo de cambio en el mercado negro de más del $20 \%$ en la década de 1970 o de 1980. RODRÍGUEZ y RODRIK (2001) muestran que la mayor parte de la correlación de este indicador con el crecimiento se debe a los dos últimos requisitos. El monopolio estatal de exportaciones que es muy común en los países africanos, y el diferencial de tipo de cambio que de hecho es un indicador de severos problemas macroeconómicos. Al interpretar los resultados que presentamos en este trabajo deberemos tener en cuenta que este indicador puede recoger en parte un componente geográfico o macroeconómico, y no tan sólo el efecto de la política de apertura.
} 
De acuerdo con este índice, el país con una política de competencia más efectiva es Finlandia con un valor de 6,6, seguido por Alemania, Holanda y Estados Unidos. En el otro extremo encontramos con el menor valor a Honduras $(2,1)$ y con valores similares a Ecuador, Guatemala y Uruguay. Debe mencionarse, que ninguno de estos últimos países cuenta con una autoridad de defensa de la competencia, ni con leyes de competencia que abarquen al conjunto de las actividades económicas. Esta medida nos indica la efectividad de la política de competencia tal como es percibida por los agentes económicos. Se toma el valor de este indicador para el año 2000.

Seguimos a Persson y Tabellini (2003) al utilizar las siguientes variables exógenas determinantes de las políticas de crecimiento: latitud (LAT01) tal y como la definen Hall y Jones (1999) e indicadores regionales (OECD, América Latina - LAAM, Asia Oriental - ASIAE y África - AFRICA); la localización geográfica y los recursos físicos están recogidos en el logaritmo de la predicción de la participación en el comercio internacional de cada país (FRANKROM) a partir de un modelo de gravitación del comercio internacional estimado por Frankel y Romer (1999); los determinantes históricos son capturados por variables como la fracción de la población que tiene el inglés como lengua materna (ENGFRAC) o que tienen uno de los cinco idiomas europeos dominantes (incluido el inglés) como lengua materna (EURFRAC) tal y como las ofrecen Hall y Jones (1999); los determinantes institucionales vienen recogidos por una variable que indica si cada país es federal o no lo es (FEDERAL), y por un conjunto de variables que señalan el origen colonial depreciado por el número de años desde la independencia (COL_UKA, COL_ESPA, COL_OTHA).

En el cuadro 1, mostramos los estadísticos descriptivos de las variables de productividad (A - output por ocupado y YL - productividad total de los factores) y de políticas (GADP - calidad institucional, YROPEN - apertura y COMP_EF - efectividad de la política de competencia) que utilizaremos en nuestro análisis. Distinguimos entre la muestra completa de 52 países y la muestra restringida que excluye a los países de América Latina. Estos países tienen en promedio una menor calidad institucional, y a su vez una mayor dispersión que como discutiremos más adelante dificulta el proceso de identificación y estimación del efecto de las políticas de competencia sobre la productividad total de los factores.

El cuadro 2 muestra la correlación entre las políticas endógenas que explican la productividad (GADP, YRSOPEN y COMP_EF). Existe una limitada correlación entre la variable de calidad institucional (GADP) y la variable de apertura (YRSOPEN). Por el contrario, existe una elevada correlación entre la calidad institucional (GADP) y la variable que mide la efectividad de la política de competencia (COMP_EF). Por ello, es difícil distinguir el efecto independiente de cada una de estas dos últimas variables sobre la productividad. 


\section{CUADRO 1 \\ ESTADÍSTICOS DESCRIPTIVOS}

\begin{tabular}{|l|c|c|c|c|c|}
\hline & $\mathrm{n}$ & Media & Desv. est. & Min & Max \\
\hline A & 52 & $4.479,10$ & $1.964,99$ & $1.156,14$ & $8.229,00$ \\
\hline YL & 52 & $16.252,03$ & $9.959,47$ & $2.317,77$ & $35.438,70$ \\
\hline GADP & 52 & 0,73 & 0,20 & 0,37 & 1,00 \\
\hline YRSOPEN & 52 & 0,54 & 0,34 & 0,00 & 1,00 \\
\hline COMP_EF & 52 & 4,49 & 1,13 & 2,10 & 6,60 \\
\hline \multicolumn{7}{|c|}{ MUESTRA RESTRINGIDA (sin América Latina) } \\
\hline \multicolumn{7}{|c|}{ n } & $\mathrm{n}$ & Media & Desv. est. & Min & Max \\
\hline A & 35 & $4.929,39$ & $2.055,82$ & $1.156,14$ & $8.229,00$ \\
\hline YL & 35 & $19.769,73$ & $10.106,54$ & $2.317,77$ & $35.438,70$ \\
\hline GADP & 35 & 0,83 & 0,16 & 0,41 & 1,00 \\
\hline YRSOPEN & 35 & 0,63 & 0,35 & 0,00 & 1,00 \\
\hline COMP_EF & 35 & 5,00 & 0,89 & 3,20 & 6,60 \\
\hline Fuente: Persson y Tabellini (2003) y Porter et al. (2000). \\
\hline
\end{tabular}

CUADRO 2

CORRELACIONES ENTRE POLÍTICAS

\begin{tabular}{|c|c|c|}
\hline & GADP & YRSOPEN \\
\hline YRSOPEN & $58 \%$ & \\
\hline COMP_EF & $89 \%$ & $40 \%$ \\
\hline \multicolumn{3}{|c|}{ MUESTRA RESTRINGIDA (sin América Latina) } \\
\hline & GADP & YRSOPEN \\
\hline YRSOPEN & $61 \%$ & \\
\hline COMP_EF & $83 \%$ & $34 \%$ \\
\hline
\end{tabular}

\section{Estimaciones y resultados}

A continuación mostramos los resultados de estimar el impacto de la calidad institucional (GADP) y de apertura (YRSOPEN) sobre la productividad. Se trata de una réplica de la estimación de Persson y Tabellini (2003) restringida en este caso a la muestra de 52 países en vez de la muestra de 74 que ellos utilizan para sus estimaciones. Se muestran los resultados de estimar las ecuaciones de output por ocupado y de productividad total de los factores por mínimos cuadrados ordinarios, así como la estimación a través de variables instrumentales que intenta reducir el conocido 
sesgo de atenuación que se deriva del carácter endógeno de las políticas: el coeficiente estimado por mínimos cuadrados se aproxima a cero, y los errores estándares se amplían llevando los tests estadísticos a no rechazar la hipótesis nula de que el coeficiente estimado es igual a cero de forma equivocada.

El cuadro 3 muestra que la calidad institucional (GADP) tiene un impacto significativo y positivo sobre la productividad total de $\operatorname{los}$ factores $(\log \mathrm{A})$ y el output por

CUADRO 3

PRODUCTIVIDAD, CALIDAD Y APERTURA

\begin{tabular}{|c|c|c|c|c|}
\hline & $\begin{array}{c}(1) \\
\mathrm{MCO} \\
\log \mathrm{A}\end{array}$ & $\begin{array}{c}(2) \\
\mathrm{VI} \\
\log \mathrm{A}\end{array}$ & $\begin{array}{c}(3) \\
\mathrm{MCO} \\
\log \mathrm{YL}\end{array}$ & $\begin{array}{c}(4) \\
\text { VI } \\
\log \mathrm{YL}\end{array}$ \\
\hline Constante & $6,38(0,30)^{* * *}$ & $6,10(0,30)^{* * *}$ & $6,34(0,36) * * *$ & $5,83(0,45)^{* * *}$ \\
\hline GADP & $2,07(0,36) * * *$ & $2,33(0,44) * * *$ & $3,78(0,36) * * *$ & $4,58(0,51)^{* * *}$ \\
\hline YRSOPEN & $0,44(0,19)^{* *}$ & $0,59(0,27)^{* *}$ & $0,27(0,17)^{* *}$ & $0,05(0,27) * * *$ \\
\hline COMP_EF & & & & \\
\hline COL_OTHA & $0,03(0,17)$ & $-0,01(0,14)$ & $0,22(0,18)$ & $0,07(0,16)$ \\
\hline LAAM & $0,47(0,18)$ & $0,60(0,17)$ & $0,58(0,19)$ & $0,77(0,22)$ \\
\hline$R^{2}$ & 0,58 & 0,55 & 0,80 & 0,78 \\
\hline Test de la F & $21,97 * * *$ & $29,62 * * *$ & $67,83 * * *$ & $56,31 * * *$ \\
\hline Test J de Hansen & & 6,80 & & 10,11 \\
\hline$n$ & 52 & 52 & 52 & 52 \\
\hline
\end{tabular}

Instrumentos de la primera etapa: latitud - LAT01, fracción de angloparlantes - ENGFRAC, fracción de hablantes de idiomas europeos - EURFRAC, país de estructura federal - FEDERAL, origen colonial británico COL_UKA, origen colonial español - COL_ESPA, país de Asia Oriental - ASIAE, país de Africa - AFRICA.

$* * *, * * \mathrm{y} *$ representan niveles de significación del 1,5 y $10 \%$ respectivamente.

ocupado (log YL). Por su parte, la apertura comercial (YRSOPEN) tiene un impacto significativo y positivo sobre la productividad total de los factores $(\log \mathrm{A})$. La magnitud de los coeficientes es muy similar a las estimaciones de Persson y Tabellini (2003). Sólo la apertura deja de ser significativa en la ecuación de output por ocupado, mientras que estos autores encuentran que es significativa al $10 \%$.

Todas las variables exógenas de carácter geográfico e histórico se utilizan como instrumentos ya que afectan a la productividad de forma indirecta, a través de las políticas. En cambio las variables América Latina (LAAM) y Origen colonial europeo diferente al anglosajón o español (COL_OTHA) afectan directamente a la productividad ya que no superan el test de validez como instrumentos. El test J de Hansen sobre la validez de los instrumentos muestra que también son válidos para el caso de la muestra de 52 países. Todas las estimaciones por variables instrumentales se realizan mediante el método generalizado de los momentos en dos etapas robusto a la presencia de heterocedasticidad.

El cuadro 4 muestra los resultados de estimar estas mismas ecuaciones de productividad incorporando el efecto de la política de competencia. La elevada correla- 
ción entre la efectividad de la política de competencia (COMP_EF) y la calidad institucional (GADP) no permite identificar ni estimar de forma precisa por separado el efecto particular de la política de competencia sobre la productividad total de los factores. En la estimación por MCO, los coeficientes que miden el efecto de las políticas sobre la productividad tienen el signo esperado, pero la política de competencia no aparece como significativa. En la estimación por variables instrumentales, el coeficiente es incluso negativo aunque no significativo. Tampoco es significativo el coeficiente de la calidad institucional ni el de la apertura.

En el caso del output por ocupado (YL), la estimación por mínimos cuadrados ordinarios sí permite distinguir el efecto positivo y significativo de las tres variables:

\section{CUADRO 4 \\ PRODUCTIVIDAD, CALIDAD INSTITUCIONAL, APERTURA Y POLÍTICA DE COMPETENCIA}

\begin{tabular}{|c|c|c|c|c|}
\hline & $\begin{array}{c}(1) \\
\mathrm{MCO} \\
\log \mathrm{A}\end{array}$ & $\begin{array}{c}(2) \\
\text { VI } \\
\log A\end{array}$ & $\begin{array}{c}(3) \\
\mathrm{MCO} \\
\log \mathrm{YL}\end{array}$ & $\begin{array}{c}(4) \\
\text { VI } \\
\log \mathrm{YL}\end{array}$ \\
\hline Constante & $6,27(0,32)^{* * *}$ & $6,09(0,39)^{* * *}$ & $6,18(0,34) * * *$ & $5,72(0,40) * * *$ \\
\hline GADP & $1,31(0,63)^{* *}$ & $2,82(1,86)$ & $2,69(0,60) * * *$ & $2,05(1,56)$ \\
\hline YRSOPEN & $0,51(0,19)^{* * *}$ & $0,48(0,47)$ & $0,37(0,18) * *$ & $0,61(0,44)$ \\
\hline COMP_EF & $0,14(0,11)$ & $-0,07(0,28)$ & $0,20(0,10)^{* *}$ & $0,38(0,21)^{*}$ \\
\hline COL_OTHA & $-0,04(0,17)$ & $-0,03(0,21)$ & $0,11(0,16)$ & $-0,11(0,17)$ \\
\hline LAAM & $0,47(0,18) * *$ & $0,61(0,20)^{* * *}$ & $0,58(0,19)^{* * *}$ & $0,74(0,21)^{* * *}$ \\
\hline$R^{2}$ & $0,0,59$ & 0,53 & 0,82 & 0,79 \\
\hline Test de la F & $18,85 * * *$ & $18,76 * * *$ & $68,88 * * *$ & $69,22 * * *$ \\
\hline Test J de Hansen & & 6,29 & & 9,03 \\
\hline$n$ & 52 & 52 & 52 & 52 \\
\hline
\end{tabular}

Instrumentos de la primera etapa: Tatitud - LAT01, fracción de angloparlantes - ENGFRA de idiomas europeos EURFRAC, FEDERAL, origen colonial británico COL_UKA, origen co COL_ESPA, país de Asia Oriental - ASIAE, país de Africa - AFRICA.

$* * *, * * \mathrm{y} *$ representan niveles de significación del 1, 5 y $10 \%$ respectivamente.

calidad institucional, apertura y política de competencia. La política de competencia parece tener un impacto diferencial sobre la productividad por ocupado. Además, al estimar la ecuación por variables instrumentales, tan sólo mantiene la significación la variable de política de competencia. La elevada correlación entre las variables explicativas parece elevar los errores estándares de manera que la calidad institucional y la apertura pierden su significatividad aunque mantienen el signo positivo esperado.

Tal y como ya hemos adelantado anteriormente, una observación detallada de los datos permite observar que los países de América Latina presentan una dispersión mayor en cuanto a la calidad institucional y a la efectividad de la política de competencia que el resto de los países de la muestra. Los gráficos 1 y 2 muestran la correlación parcial entre la productividad total de los factores $(\log$ A) y la efectivi- 


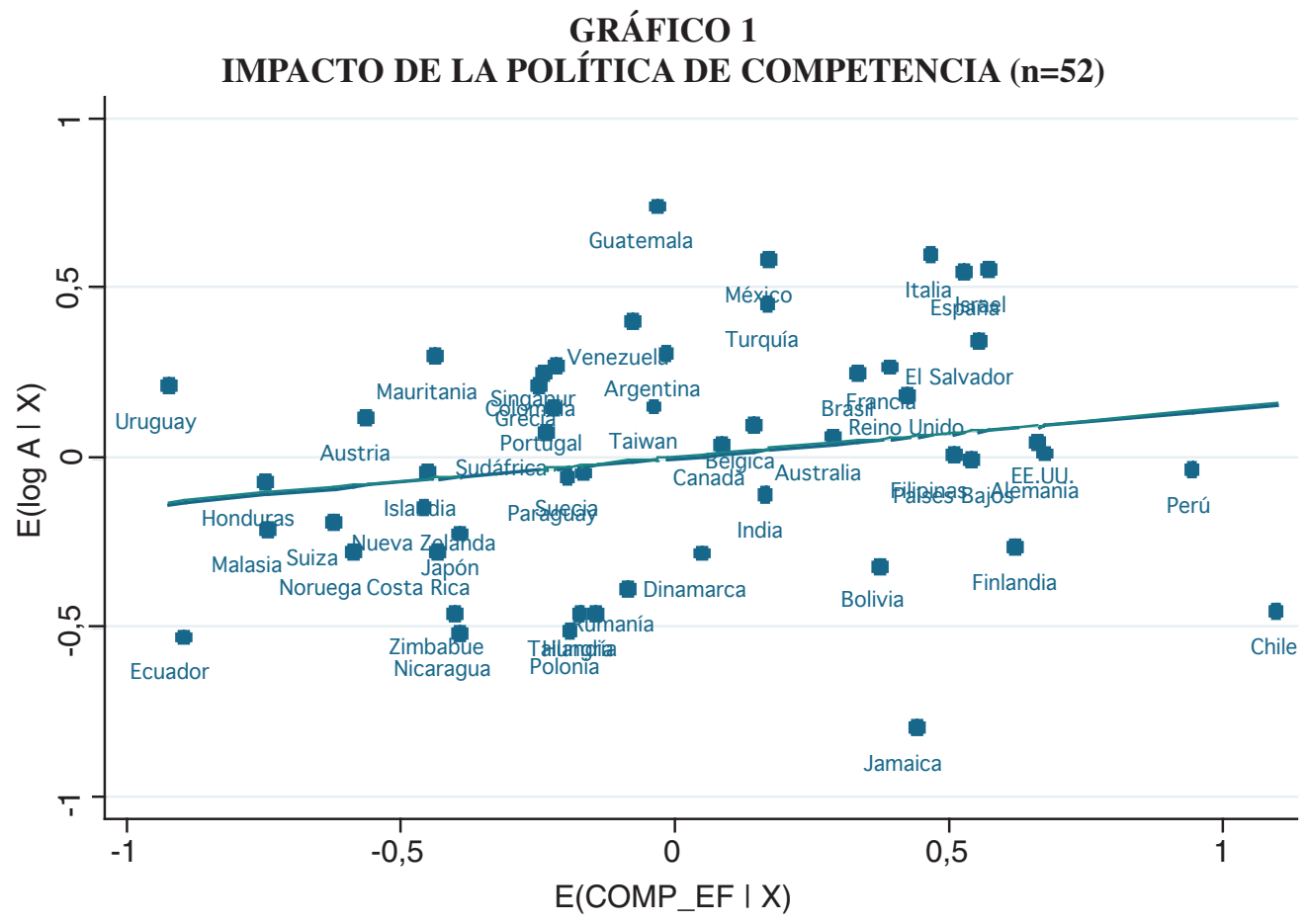

coeficiente $=0,14$, error estándar robusto $=0,11, t=1,34$

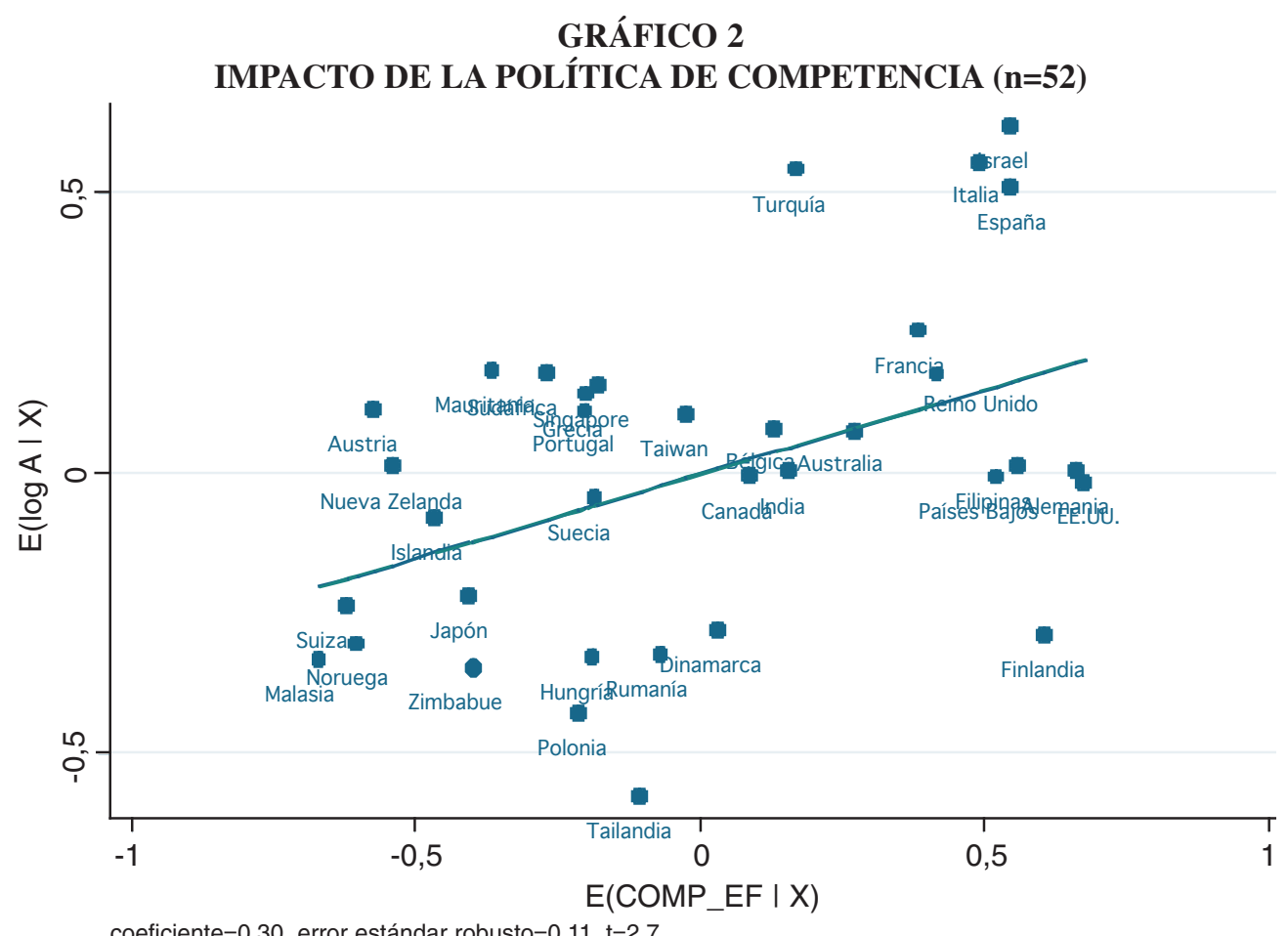

coeficiente $=0,30$, error estándar robusto $=0,11, t=2,7$ 
dad de la política de competencia (COMP_EF) para la muestra completa $(\mathrm{n}=52)$, y para la muestra restringida $(n=35)$. En el gráfico 1 se observa que los países de América Latina se sitúan mayoritariamente en los extremos más alejados de la recta de regresión en todos los cuadrantes.

Esta elevada dispersión puede estar dificultando el proceso de identificación y estimación del efecto de estas variables sobre la productividad. A pesar de que eliminar los países de América Latina reduce la muestra a sólo 35 países, es interesante comprobar en qué medida los resultados son distintos si consideramos sólo la muestra restringida de países para los que existe una mayor uniformidad en cuanto al grado de calidad institucional. El gráfico 2 muestra la mayor correlación parcial entre la productividad total de los factores $(\log$ A) y la efectividad de política de competencia (COMP_EF) cuando no tenemos en cuenta a los países de América Latina.

El cuadro 5 muestra que para esta muestra restringida de países con un nivel más homogéneo de calidad institucional, las diferencias en la efectividad de la política de competencia importa. En el caso de la productividad total de los factores, la política de competencia (COM_EF) tiene un impacto significativo y positivo. La variable de apertura (YRSOPEN) mantiene la significatividad y el signo positivo. Por el contrario, la calidad institucional pierde su significación, a pesar de que mantiene el signo positivo. La estimación de este impacto positivo es robusta a la utilización de variables instrumentales.

En el caso del output por ocupado, la política de competencia (COMP_EF) tiene un impacto positivo y significativo. En la estimación por mínimos cuadrados ordinarios, se mantiene la significatividad y el signo positivo de las tres variables: la calidad institucional (GADP), la apertura (YRSOPEN) y la política de competencia (COMP_EF). En el caso de la estimación por variables instrumentales, la calidad institucional pierde completamente su significatividad y se torna negativa. El cuadro muestra que las ecuaciones superan el test de Hansen de validez de los instrumentos, no se rechazan las condiciones de sobreidentificación.

En definitiva, con las estimaciones anteriores hemos sido capaces de mostrar la elevada correlación parcial entre la efectividad de la política de competencia (COM_EF) y el output por ocupado (log YL), así como entre la política de competencia (COMP_EF) y la productividad por total de los factores $(\log \mathrm{A})$ en el caso de los países con un nivel de calidad institucional suficientemente elevado. Esta correlación es significativa incluso en numerosas estimaciones a través de mínimos cuadrados ordinarios que como se sabe sufren un sesgo de atenuación (es decir, una reducción del valor medio de los coeficientes y ampliación de los errores estándares) cuando las variables explicativas son endógenas.

Las estimaciones por variables instrumentales sufren dificultades a la hora de corregir este sesgo de atenuación debido a que nuestros instrumentos no son suficientemente fuertes. El cuadro 6 muestra que ni en el caso de la muestra de 52 países, ni en la de 35, los instrumentos superan el test de Stock y Yogo (2005) que permite rechazar la hipótesis nula de que el sesgo de las estimación por variables 


\section{CUADRO 5 \\ PRODUCTIVIDAD, CALIDAD INSTITUCIONAL, APERTURA Y POLÍTICA DE COMPETENCIA (AMÉRICA LATINA EXCLUIDA)}

\begin{tabular}{|c|c|c|c|c|}
\hline & $\begin{array}{c}(1) \\
\mathrm{MCO} \\
\log \mathrm{A} \\
\end{array}$ & $\begin{array}{c}(2) \\
\text { VI } \\
\log \mathrm{A} \\
\end{array}$ & $\begin{array}{c}(3) \\
\mathrm{MCO} \\
\log \mathrm{YL} \\
\end{array}$ & $\begin{array}{c}(4) \\
\text { VI } \\
\log \mathrm{YL} \\
\end{array}$ \\
\hline Constante & $6,20(0,31)^{* * *}$ & $5,95(0,25) * * *$ & $5,99(0,36) * * *$ & $5,72(0,33) * * *$ \\
\hline GADP & $0,17(0,68)$ & $0,42(0,87)$ & $1,86(0,69) * *$ & $-0,29(0,76)$ \\
\hline YRSOPEN & $0,89(0,21) * * *$ & $0,91(0,25)^{* * *}$ & $0,57(0,22) * *$ & $0,89(0,34)^{* * *}$ \\
\hline COMP_EF & $0,30(0,11)^{* * *}$ & $0,30(0,15)^{*}$ & $0,36(0,11) * * *$ & $0,74(0,14)^{* * * *}$ \\
\hline COL_OTHA & $-0,08(0,11)$ & $-0,09(0,15)$ & $0,06(0,15)$ & $-0,18(0,14)$ \\
\hline LAAM & & & & \\
\hline$R^{2}$ & 0,79 & 0,78 & 0,88 & 0,83 \\
\hline Test de la F & $21,75^{* * *}$ & $109,8 * * *$ & $59,01 * * *$ & $113,34 * * *$ \\
\hline Test $J$ de Hansen & & 9,51 & & 10,56 \\
\hline$n$ & 35 & 35 & 35 & 35 \\
\hline
\end{tabular}

Instrumentos de la primera etapa: latitud - LAT01, fracción de angloparlantes - ENGFRAC, fracción de hablantes de idiomas europeos - EURFRAC, país de estructura federal - FEDERAL, origen colonial británico COL_UKA, origen colonial español - COL_ESPA, país de Asia Oriental - ASIAE, país de Africa - AFRICA.

$* * *, * * \mathrm{y} *$ representan niveles de significación del 1, 5 y $10 \%$ respectivamente.

instrumentales es como máximo un 30\% el sesgo de la estimación por mínimos cuadrados ordinarios.

Con todo, en la muestra reducida de 35 países, los instrumentos parecen ser incluso más fuertes que en el caso de la muestra de 52 países: el $\mathrm{R}^{2}$ parcial y el test de F son sustancialmente mayores. Nuestros instrumentos tienen especial dificultad en predecir la varianza en la variable apertura, ya que para esta variable el test de la F es inferior a 10. Por ello, no podemos hacer una interpretación causal de los parámetros estimados, pero sí concluir que existe una elevada correlación parcial en los casos en los que los parámetros son significativos incluso cuando los coeficientes estimados puedan estar atenuados y los errores estándares sean demasiado elevados por la presencia de endogeneidad no corregida.

\section{Conclusión}

En este trabajo, hemos comprobado la dificultad de separar el impacto de una política concreta como es la política de competencia sobre la productividad total de los factores y el output por ocupado, del impacto positivo de la calidad en el entorno institucional y la apertura utilizando datos a nivel de país.

Tal y como ya habían puesto de manifiesto trabajos anteriores como Voigt (2006), las estimaciones para un amplio conjunto de países que contienen variables que indican la calidad diferencial de las políticas impide estimar el efecto separado 


\begin{tabular}{|c|c|c|}
\hline \multicolumn{3}{|c|}{$\begin{array}{c}\text { CUADRO } 6 \\
\text { FORTALEZA DE LOS INSTRUMENTOS }\end{array}$} \\
\hline & $\begin{array}{l}\text { Muestra } \\
\text { completa } \\
(n=52)\end{array}$ & $\begin{array}{c}\text { Muestra } \\
\text { restringida } \\
(\mathrm{n}=35)\end{array}$ \\
\hline \multicolumn{3}{|l|}{$R^{2}$ parcial de los instrumentos excluidos } \\
\hline GADP & 0,48 & 0,63 \\
\hline YRSOPEN & 0,30 & 0,40 \\
\hline COMP_EF & 0,52 & 0,60 \\
\hline \multicolumn{3}{|l|}{ Test de la $F$ de los instrumentos excluidos } \\
\hline GADP & $9,45 * * *$ & $17,95 * * *$ \\
\hline YRSOPEN & $2,87 * *$ & $9,27 * *$ \\
\hline COMP_EF & $21,84 * * *$ & $21,84 * * *$ \\
\hline Test de debilidad de los instrumentos & 3,06 & 2,85 \\
\hline
\end{tabular}

***, ** y* representan niveles de significación del 1, 5 y $10 \%$ respectivamente.

de políticas como la competencia. En este trabajo, hemos comprobado que la utilización de técnicas de variables instrumentales permiten superar sólo parcialmente este problema.

Sin embargo, hemos sido capaces de identificar la correlación parcial que existe entre la efectividad de las políticas de competencia y el output por ocupado, así como entre la efectividad de la política de competencia y la productividad total de los factores si restringimos el análisis a países que no se distancian mucho en cuanto la calidad del entorno institucional.

Los resultados obtenidos nos permiten concluir que la política de competencia está asociada al mejor uso del factor trabajo, y con el uso de la innovación en productos y procesos en países que gozan de entornos institucionales de calidad. Con todo, los efectos de la política de competencia pueden ser muy diversos entre países. América Latina parece tener dificultades en trasladar la efectividad de la política de competencia en ganancias de productividad total de los factores.

Queda pendiente para trabajos posteriores la utilización de técnicas que permitan mejorar las estimaciones en muestras para las que se disponen de instrumentos débiles como las propuestas por Hahn y Asuman (2003) y Andrews y Stock (2007). Estas técnicas deberían permitir precisar más el papel de la política de competencia en la mejora de la productividad de los países. 


\section{Referencias}

[1] AI, C. y D. E. M. SAPPINGTON (2002). «The Impact of State Incentive Regulation on the US Telecommunications Industry», Journal of Regulatory Economics, 22: 2, 133 160.

[2] ANDREWS, D. W. y J. H. STOCK (2007). «Inference with Weak Instruments, in Advances in Economics and Econometrics: Theory and Applications», Econometric Society Ninth World Congress Proceedings, vol. III, R. Blundell, W. Newey y T. Persson (eds.). Cambridge University Press.

[3] ACEMOGLU, D., S. JONSON y J. A. ROBINSON (2001). «The colonial origins of comparative development: an empirical investigation», American Economic Review, 91(5), 1369-1401.

[4] AGHION, P. y M. SCHANKERMAN (2004). «On the welfare effects and political economy of competiton-enhancing policies», The Economic Journal, 114, 800-824.

[5] BEN-DAVID, D. (1993). «Equalizing Exchange: Trade Liberalization and Income Convergence», Quarterly Journal of Economics, 108(3), 653-79.

[6] BESLEY T. y CASE A. (2000). «Unnatural Experiments? Estimating the incidence of endogenous policies», Economic Journal, 11, 672-694.

[7] BORRELL, J. R. y M. TOLOSA (2008). «Endogenous Antitrust: Cross-Country Evidence on the Impact of Competition-Enhancing Policies on Productivity», Applied Economics Letters, 15, 827-831.

[8] DOLLAR, D. (1992). «Outward-Oriented Developing Economies Really Do Grow More Rapidly: Evidence form 95 LDCs, 1976-85», Economic Development and Cultural Change, 523-544.

[9] DUSO, T. y L. H. RÖLLER (2003). «Endogenous Deregulation: Evidence from OECD Countries», Economic Letters, 81, 67-71.

[10] DUSO, T. (2005). «Lobbying and Regulation in a Political Economy: Evidence from the U.S. Cellular Industry», Public Choice, 122: 3-4, 251-276.

[11] EASTERLY, W. y R. LEVINE (2003). «Tropics, Germs, and Crops», Journal of Monetary Economics, 50, 3-39.

[12] EDWARDS, S. (1998). «Openness, Productivity and Growth: What Do We Really Know», Journal of Development Economics, 39(1), 31-57.

[13] FRANKEL, J. A. y D. ROMER (1999). «Does Trade Cause Growth?», American Economic Review, 89(3), 379-399.

[14] HALL, R. y C. JONES (1999). «Why some countries produce so much more output per worker than others?», Quarterly Journal of Economics, 114, 83-116.

[15] HAHN, J. y J. HAUSMAN (2003). «Weak Instruments: Diagnosis and Cures in Empirical Econometrics», American Economic Review, 93(2), 118-125.

[16] HAYRI A. y M. DUTZ (1999). «Does more Intense Competition lead to Higher Growth?», CEPR Discussion Paper No. 2249.

[17] KEE, H. L. y B. HOEKMAN (2007). «Imports, entry and competition law as market disciplines», European Economic Review, 51, 831-858.

[18] KRAKOWSKI, M. (2005). «Competition Policy Works: The Effect of Competition Policy on the Intensity of Competition - An International Cross-Country Comparison», HWWA Discussion Paper, 332, Hamburg.

[19] McClOUGHAN, P.; S. LYONS y W. BATT (2007). «The Effectiveness of Competi- 
tion Policy and the Price-Cost Margin: Evidence from Panel Data», ESRI Working paper.

[20] PERSSON, T. y G. TABELLINI (2003). The Economic Effects of Constitutions, MIT Press, Cambridge (MA) y Londres.

[21] PRZYBYLA, M. y M. ROMA (2005). «Does Product Market Competition Reduce Inflation? Evidence form EU Countries and Sectors», European Central Bank Working Paper, 453, Frankfurt.

[22] RODRÍGUEZ, F. y D. RODRIK (2001). «Trade policy and economic growth: A skeptic's guide to the cross-national evidence», NBER Macroeconomics Annual 2000, 15, 261-325.

[23] RODRIK, D.; A. SUBRAMANIAN y F. TREBBI (2004). «Institutions rule: the primacy of institutions over geography and integration in economic development», Journal of Economic Growth, 9(2), 131-65.

[24] SACHS, J. y A. WERNER (1995). «Economic reform and the process of global interpretation», Brookings Papers on Economic Activity, 1, 1-95.

[25] SACHS J. (2003). «Institutions don't rule: direct effects of geography on per capita income», NBER Working Paper 9490.

[26] STOCK, J.H. y YOGO, M. (2005). «Testing for Weak Instruments in Linear IV Regression», en D.W.K. Andrews y J.H. Stock (eds.), Identification and Inference for Econometric Models: Essays in Honor of Thomas Rothenberg, Cambridge: Cambridge University Press, pp. 80-108.

[27] SYMEONIDIS, G. (2000). «Price Competition and Market Structure: the Impact of Cartel Policy on Concentration in the UK», Journal of Industrial Economics, 48, 1-26.

[28] SYMEONIDIS, G. (2008). «The Effect of Competition on Wages and Productivity: Evidence from the UK», Review of Economics and Statistics, 90, 134-146.

[29] PORTER M. et al. (2000). The World Competitiveness Report by the World Economic Forum and Harvard University, Oxford University Press, Oxford.

[30] VOIGT, S. (2006). «The economic effects of competition policy: cross-country evidence using four new indicators», Social Science Research Network Working Paper, No. 925794. Disponible en http://ssrn.com/abstract=925794. 